\title{
Quercus Brantii Lindl. Vaginal Douche Versus Clotrimazole on Vaginal Candidiasis
}

\section{- A Randomized Clinical Trial -}

\author{
Zeinab Moshfeghy ${ }^{1,2 *}$, Khadegeh Asadi ${ }^{2}$, Marzieh Akbarzadeh ${ }^{3}$, Atefeh Zare ${ }^{2}$, Ta- \\ hereh Poordast ${ }^{4,5}$, Masoumeh Emamghoreishi ${ }^{6}$, Fatemeh S.Najib ${ }^{4,5}$, Mehrab Saya- \\ $\mathrm{di}^{7}$
}

${ }^{1}$ Community Based Psychiatric Care Center, Shiraz University of Medical Sciences, Shiraz, Iran

2 Department of Midwifery, School of Nursing and Midwifery, Shiraz University of Medical Sciences, Shiraz, Iran

${ }^{3}$ Maternal-Fetal Research Center, Department of Midwifery, Shiraz University of Medical Sciences, Shiraz, Iran

${ }^{4}$ Infertility Research Center, Shiraz University of Medical Science, Shiraz, Iran

${ }^{5}$ Department of OB/GYN, School of Medicine, Shiraz University of Medical Sciences, Shiraz, Iran

${ }^{6}$ Department of Pharmacology, School of Medicine, Shiraz University of Medical Sciences, Shiraz, Iran

${ }^{7}$ Cardio-vascular Research Center, Shiraz University of Medical Sciences, Shiraz, Iran

\section{Key Words}

clotrimazole vaginal cream, extract, Quercus Brantii Lindl., Vaginal Candidiasis, vaginal douche

\begin{abstract}
Objectives: Vaginal Candidiasis with an approximate prevalence of $30 \%$ is the second cause of vaginal infections. Antifungal azole is the first treatment for Vaginal Candidiasis; however, some side effects have been reported for this chemical medicine. Based on the antifungal activity of Inner Stratum of Quercus Brantii ( $Q$. Brantii), the aim of our study was to compare the effects of vaginal douche of Q. Brantii extract and clotrimazole on vaginal candidiasis symptoms before and after the treatments, in women.
\end{abstract}

Methods: 89 non-pregnant women with positive $\mathrm{KOH}$ test which is capable of identifying the presence of hyphae and mycelium by adding $\mathrm{KOH}(10 \%)$, and a positive vaginal candidiasis culture were randomly divided into two experimental groups, using permuted block randomization method. One group received clotrimazole vaginal cream (1\%) and the other group received

Received: Feb 11, 2018 Reviewed: Jul 11, 2018 Accepted: Aug 02, 2018

(1) This is an Open-Access article distributed under the terms of the Creative Common Attribution Non-Commercial License (http://creativecommons.org/licenses/by-nc/4.0/) which permits unrestricted noncommercial use, distribution, and reproduction in any medium, provided the original work is properly cited.

(2) This paper meets the requirements of KS X ISO 9706, ISO 9706-1994 and ANSI/NISO Z39.48-1992 (Permanence of Paper). vaginal douche of $Q$. Brantii extract. Groups were treated for 7 days and $\mathrm{KOH}$ tests and cultures were evaluated again. Data were analyzed via chi-square and independent t-test, using SPSS software.

Results: According to the results, there were no significant differences between experimental groups for demographic characteristics like age $(\mathrm{p}=0.403), \mathrm{BMI}$ $(\mathrm{p}=0.911)$, educational levels $(\mathrm{p}=0.862)$ and contraceptive methods $(\mathrm{p}=0.702)$. Moreover, significant differences were seen in vaginal discharge between the groups after the treatments $(\mathrm{P}=0.043)$.

Conclusion: The results suggested that the therapeutic effect of vaginal douche of $Q$. Brantii extract was approximately similar to that of clotrimazole vaginal cream.

\section{Introduction}

Vaginal candidiasis is one of the most frequent infections of the female genital tract [1]. Vaginal candidiasis is the second most common cause of vaginitis worldwide, after the bacterial infection. The risk of vaginal candidiasis for non-pregnant women is approximately $20 \%$, but it increases by $30 \%$ during pregnancy [2]. No complete reporting of the number of women involved

"Corresponding Author

Zeinab Moshfeghy. Community Based Psychiatric Care Research Center, Department of Midwifery, School of Nursing and Midwifery, Shiraz University of Medical Sciences, Shiraz, Iran.

Tel: +98-713-647-4256 Fax: +98-713-647-4252

E-mail: zmoshfeghy1385@yahoo.com 
in Candida vaginitis is available in Iran [3], but according to a study, the prevalence of vaginal candidiasis in Iran is between $25 \%$ and $45 \%$ [4]. The main reasons for the recurrence of Candida are not fully understood [5]. Autoimmune diseases, endocrine diseases, diabetes mellitus, and antibiotic therapy are the main causes of vaginal candidiasis [6]. The typical symptoms of vaginal candidiasis include pruritus, vaginal soreness, dyspareunia, external dysuria, and abnormal vaginal discharge [7]. Patients may be occasionally found to have candida based on a culture or Pap smear [8]. Vulvovaginal candidias is as an acute inflammatory disease, caused by Candida sp. especially $C$. albicans through non-albicans species of Candida (C.glabrata, C.tropicalis and C.dubliniensis) are also associated with recurrent diseases. Candidiasis is associated with intense pruritus, itching, dyspareunia, erythematous vulva, and cottage cheese like discharges, positive vaginal smear and culture, and a vaginal $\mathrm{pH}$ of less than 4.5. However, non-albicans species are usually less sensitive to azole antifungal agents $[9,10]$. Antifungal azole drugs provide the first treatments for vaginal candidiasis. Nevertheless, some side effects have been reported for this chemical medicine. Over-the-counter (OTC) of miconazole and clotrimazole medications for 7 days would provide a proper first-line therapy for women with occasional yeast vaginitis [11].

Several topical and oral antifungals such as clotrimazole is used for the treatment of vaginal candidiasis [12]. Nevertheless, some side effects, such as changes in hepatic enzymes, painful urination, depression, irritation, and dermatitis have been reported for clotrimazole [13]. Although it has no side effects during pregnancy, it is associated with embryotoxic effects. Thus, it should be consumed with caution during breast-feeding [14]. Also, the long-term use of antifungal drugs would lead to recurrent vaginitis [11]. Unavailability and high costs of the drugs in low-income societies, as well as their probable side effects and resistances have resulted in increased worries about synthetic chemicals [15]. In recent years, people have shown a growing interest in the general trends of natural remedies and herbal therapies when considering the lower costs, high availabilities, and lower side effects of plants and plantbased chemicals [16].

Since long time ago, plants have been used in traditional medicine practiced in almost all Asian countries including Iran. Herbal medicine still plays an essential role in various therapeutic fields, though synthetic applications of drugs in modern medicine have advanced over the past decades. In fact, the interest in herbal medications has been raised due to the therapeutic properties of plants. Several studies have revealed that plants have great amounts of natural antioxidants, as well as large anti-inflammatory [17], antibacterial [18], and antiviral [19] contents that make them highly important in the different aspects of medical applications. The fruit of Quercus brantii Lindl. is a source of tannins, catechins, gallic and ellagic acids, different galloyl and hexahydroxydiphenoyl derivatives, and oligomeric and polymeric proanthocyanidins [20]. Alcoholic Extract of Inner Stratum of $Q$. Brantii Fruit has antifungal effects on Candida albicans [21]. According to the literature, there are no reports on the effect of vaginal douche of Inner Stratum of $Q$. Brantii on vaginal candidiasis. Thus, the current investigation aimed at comparing the effects of two treatments, including vaginal douche of $Q$. Brantii extract and clotrimazole vaginal cream (1\%) on vaginal candidiasis symptoms before and after the treatments in women.

\section{Materials and Methods}

\subsection{Patients}

This research was a randomized, case-controlled clinical trial. In this study, 89 non-pregnant women, who had a positive $\mathrm{KOH}$ test and culture of vaginal candidiasis and had been referred to the gynecology clinics of Shiraz University of Medical Sciences during 2016 were included into the study. To select the samples, convenience sampling were utilized and then we divided the patients into 2 groups based on the inclusion criteria of being married and having an age of 18-49 years and symptoms of vaginal candidiasis, respectively [22]. All the women were investigated for vaginal edema and erythema and other clinical symptoms of vaginal candidiasis. Also, only single sexual partners married to their respective spouses were chosen [3]. Other inclusion criteria were: having no abnormal vaginal or uterine bleeding, no known sensitivity to clotrimazole or other herbal drugs including Q. Brantii or known autoimmune diseases. Those women who were pregnant, had pelvic inflammatory diseases and lactation during the study, had menstruated during the study, and had taken hormone therapy, vaginal creams at least 7 days before the study, and anti-inflammatory, anti-fungal, and immunosuppressive drugs 14 days before the study were excluded from the research. All the patients signed an agreement form before participating in the investigation and after being informed about the study procedure [3]. The two codes of $\mathrm{A}$ and $\mathrm{B}$, which respectively represented the treatments with clotrimazole vaginal cream (1\%) and vaginal douche of $Q$. Brantii, were written on small pieces of paper and put into the relevant patients' pockets. The women were randomly divided into 2 experimental groups by permuted block randomization method. Group A $(\mathrm{n}=45)$ received clotrimazole vaginal cream $(1 \%)$ and group B $(n=44)$ received vaginal douche of $Q$. Brantii. The women in group A used a clotrimazole vaginal cream (1\%) via a vaginal drug applicator for 7 nights, while those in group B dissolved the extract of $Q$. Brantii in $150 \mathrm{cc}$ of distilled water to use vaginal douche of $Q$. Brantii for 7 nights. They were informed to lie on their backs for 30 minute after applying both the vaginal cream and douche.

\subsection{Drugs and medications}

Q. Brantii was collected from Kohgiluyeh and Boyer-Ahmad province, Iran, during 2015 and the inner stratum of the fruit was separated. The sample was authenticated and specified by with a voucher number at department of Phytopharmaceuticals, School of Pharmacy, Shiraz University of Medical Sciences. Clotrimazole vaginal cream 1\% (Mycelex) was purchased, from Pars Daru Pharma- 
ceutical Co., Tehran, Iran.

\subsection{Extraction procedure}

After collecting the fresh Q. Brantii fruit (100g), it was shade-dried at room temperature $\left(25 \pm 2^{\circ} \mathrm{C}\right)$ far from the sunlight and grounded in the Pharmacology Laboratory of Shiraz University of Medical Sciences, Shiraz, Iran. Then, the obtained powder was statically extracted in $1000 \mathrm{ml}$ of a hydroalcoholic solvent $(70 \%)$ for 72 hours at $25 \pm 2^{\circ} \mathrm{C}$. The yielded extract was filtered, concentrated and dried by vacuum evaporation. According to previous studies the extract's concentration was determined 5\% [23], and the final extract with the concentration of $5 \%$ was dissolved in the water and was used as vaginal douche every night.

\subsection{Study protocol}

Those women referred to gynecology clinics of Shiraz University of Medical Sciences and having possible symptoms of vaginal candidiasis including: itching, vaginal discharge, dysuria, dyspareunia were selected for vaginal sampling. Before initiating the study and after taking the treatment procedures, a dry speculum was inserted into the vagina and the vaginal discharge from the posterior fornix were placed on a glass slide for performing the $\mathrm{KOH}$ test (evaluation of hyphae and mycelium by adding $\mathrm{KOH} \mathrm{10 \% )} \mathrm{and} \mathrm{on} \mathrm{a} \mathrm{Sabouraud's} \mathrm{dextrose} \mathrm{agar} \mathrm{plate} \mathrm{for}$ culture of vaginal candidiasis and counting the colonies, respectively. The patients were informed not to have sexual intercourse at least for 48 hours or clean their vaginas 72 hours before sampling. Sampling of all the patients was done during the follicular phase. The clinical symptoms, such as itching, vaginal discharge, dyspareunia, and dysuria reported by patients were compared before and after the treatments.

\subsection{Laboratory analysis}

The vaginal discharge samples were cultured in an incubator for yeast detection. By using a sterile cotton swap, the discharges were placed on the plates with media selective for Candidiasis growth (Sabouraud's dextrose agar). An additional sample of vaginal discharge was taken with another swap and rolled in a glass slide for determining the presence of hyphae and mycelium by adding $\mathrm{KOH} 10 \%$. The results of the slides and cultures and colony counts were reported by an expert pathologist of Shiraz University of Medical Sciences.

\subsection{Statistical analysis}

The data of the samples were described, using descriptive statistics, which was presented as the mean \pm SD for continuous and numbers (\%) for categorical data. For analyzing both treatments, Independent t-test, and Chi-square test were used. $\mathrm{P}<0.05$ was considered to indicate significant differences between the treatments. For data analysis, IBM SPSS Statistics for Windows, Version 22.0. Armonk, NY: IBM Corp. was used.

\section{Results}

All of the participants in both two groups completed the study. The demographic characteristics of the patients are presented in Table 1. According to the data, no significant differences were observed between the patients' distributions of the groups treated with clotrimazole vaginal cream and vaginal douche of $Q$. Brantii based on age, BMI, educational levels and contraceptive methods.

In both groups, most of the patients had used withdrawal or condoms, while the fewest of them had used IUD for contraception. The lowest educational levels of the patients were either an uneducated or high school level.

The results of the presence of patients' clinical symptoms of vaginal candidiasis are presented in Table 2 . The prevalence of vaginal discharge was $90 \%$ in this study. No significant differences were discovered between the patients' vaginal discharge of the two groups before the treatments $(\mathrm{P}>0.999)$. However, vaginal discharge was significantly decreased in the group treated with vaginal douche of $Q$. Brantii compared to the group treated with clotrimazole vaginal cream after the treatment $(\mathrm{P}=0.043)$. Furthermore, no significant differences were observed between the groups regarding volvuvaginal itching as reported by patients, before $(\mathrm{P}=0.55)$ and after the treatments $(\mathrm{P}=0.57)$.

Also, no significant differences were seen between the groups in terms of dysuria $(\mathrm{P}>0.999)$, dyspareunia $(\mathrm{P}=0.823)$ and vaginal edema and erythema $(\mathrm{P}>0.999)$ after the treatments.

Based on the evidence of the patients' cultures of vaginal discharge (Table 3), no significant differences were seen between the groups considering the positive and negative culture growth, before and after the treatments $(\mathrm{P}=0.251)$.

As can be observed in Table 4, there were no significant differences between the colony counts of the patients' cultures in the two groups of clotrimazole vaginal cream and vaginal douche of before $(\mathrm{P}=0.188)$ and after the treatments $(\mathrm{P}=0.850)$.

\section{Discussion}

Infection of the lower genital tract is one of the principal reasons for providing gynecological care for women [24]. Due to the importance and association of vaginitis with Sexually Transmitted Diseases (STDs), several researches have been done with better and more comprehensive options of treatment [25]. The availability of a wide spectrum of therapeutic options has led to the discovery of the principal causes of vaginitis [26]. Despite the fact that chemical drugs are widely utilized for the treatment of infections, side effects, resistance, and high economic costs are the main limiting factors of these drugs [27]. Considering the problems of side effects and drug resistance, there is a current growing interest in complementary and alternative medicine (CAM) in general [28]. In this regard, Ebrahimi et al. [29] studied the antimicrobial effects of various agents of oak fruit extract against Escherichia coli bacteria and reported that the extract has acceptable antimicrobial 
Table 1 The demographic characteristics of participants

\begin{tabular}{|c|c|c|c|}
\hline Characteristics & $\begin{array}{l}\text { Clotrimazole vaginal cream } \\
\qquad(\mathrm{n}=\mathbf{4 0})\end{array}$ & $\begin{array}{l}\text { Vaginal douche of } Q . \text { Brantii } \\
\qquad(\mathrm{n}=40)\end{array}$ & P-value \\
\hline Age $($ Mean \pm SD $)$ & $33.65 \pm 8.28$ & $35.70 \pm 8.28$ & 0.403 \\
\hline BMI $($ Mean \pm SD $)$ & $23.65 \pm 4.90$ & $23.45 \pm 4.20$ & 0.911 \\
\hline $\begin{array}{l}\text { Educational level } \\
(\%)\end{array}$ & & & 0.862 \\
\hline Un-educated & $5(12.5)$ & $7(17.5)$ & \\
\hline Primary & $9(22.5)$ & $5(12.5)$ & \\
\hline Secondary & $7(17.5)$ & $7(17.5)$ & \\
\hline High school & $4(10)$ & $3(7.5)$ & \\
\hline Diploma & $8(20)$ & $10(25)$ & \\
\hline Academic & $7(17.5)$ & $8(20)$ & \\
\hline $\begin{array}{l}\text { Contraceptive } \\
\text { methods (\%) }\end{array}$ & & & 0.702 \\
\hline Ocp & $5(12.5)$ & $5(12.5)$ & \\
\hline Condom & $9(22.5)$ & $7(17.5)$ & \\
\hline IUD & $4(10)$ & $3(7.5)$ & \\
\hline Injections & $4(10)$ & $6(15)$ & \\
\hline Tubectomy & $3(7.5)$ & $7(17.5)$ & \\
\hline Withdrawal & $12(30)$ & $11(27.5)$ & \\
\hline Other & $3(7.5)$ & $1(2.5)$ & \\
\hline
\end{tabular}


Table 2 The patients' clinical symptoms of both groups before and after the treatment, N (\%)

\begin{tabular}{|c|c|c|c|}
\hline Variables & $\begin{array}{l}\text { Clotrimazole vaginal cream } \\
\qquad(\mathrm{n}=40)\end{array}$ & $\begin{array}{l}\text { Vaginal douche of } Q \text {. Brantii } \\
\qquad(\mathrm{n}=40)\end{array}$ & P-value \\
\hline \multicolumn{4}{|c|}{ Vaginal discharge } \\
\hline Before & $36(90)$ & $36(90)$ & $>0.999$ \\
\hline after & $8(20)$ & $2(5)$ & 0.043 \\
\hline \multicolumn{4}{|c|}{ volvuvaginal itching } \\
\hline Before & $24(60)$ & $28(70)$ & 0.552 \\
\hline after & $7(17.5)$ & $9(22.5)$ & 0.570 \\
\hline \multicolumn{4}{|l|}{ dysuria } \\
\hline Before & $23(57.5)$ & $23(57.5)$ & $>0.999$ \\
\hline after & $11(27.5)$ & $13(32.5)$ & $>0.999$ \\
\hline \multicolumn{4}{|l|}{ Dysparounia } \\
\hline Before & $19(47.5)$ & $18(45)$ & 0.823 \\
\hline After & $10(25)$ & $11(27.5)$ & 0.823 \\
\hline \multicolumn{4}{|c|}{ Edema and erythma } \\
\hline Before & $23(57.5)$ & $23(57.5)$ & $>0.999$ \\
\hline After & $0(0)$ & $0(0)$ & - \\
\hline
\end{tabular}

Table 3 The evidence of the patients' cultures of vaginal discharge between the groups before and after the treatment, $\mathrm{N}(\%)$

\begin{tabular}{|c|c|c|c|}
\hline & $\begin{array}{l}\text { Clotrimazole vaginal cream } \\
\qquad(n=40)\end{array}$ & $\begin{array}{l}\text { Vaginal douche of } Q \text {. Brantii } \\
\qquad(n=40)\end{array}$ & P-value \\
\hline Culture growth & \multicolumn{2}{|c|}{ Before the treatments } & \\
\hline positive & $40(100)$ & $40(100)$ & \\
\hline negative & $0(0)$ & $0(0)$ & - \\
\hline \multicolumn{4}{|c|}{ After the treatments } \\
\hline positive & $5(12.5)$ & $10(25)$ & 0.251 \\
\hline negative & $35(87.5)$ & $30(75)$ & \\
\hline
\end{tabular}


Table 4 The colony counts of patients' cultures in both groups, N (\%)

\begin{tabular}{lccc}
\hline & Clotrimazole vaginal cream & Vaginal douche of $Q$. Brantii & P-value \\
\cline { 2 - 4 } $\begin{array}{c}\text { Colony } \\
\text { counts }\end{array}$ & \multicolumn{3}{c}{ Before the treatments } \\
$0-100$ & $2(5)$ & $0(0)$ & \\
$101-200$ & $12(30)$ & $8(20)$ & 0.188 \\
$201-300$ & $26(65)$ & $32(80)$ & \\
& & & \\
$0-100$ & $35(87.5)$ & $30(75)$ & \\
$101-200$ & $4(10)$ & $9(22.5)$ & \\
$201-300$ & $1(2.5)$ & $1(2.5)$ & \\
\hline
\end{tabular}




\section{Enrollment}

Assessed for eligibility $(n=270)$

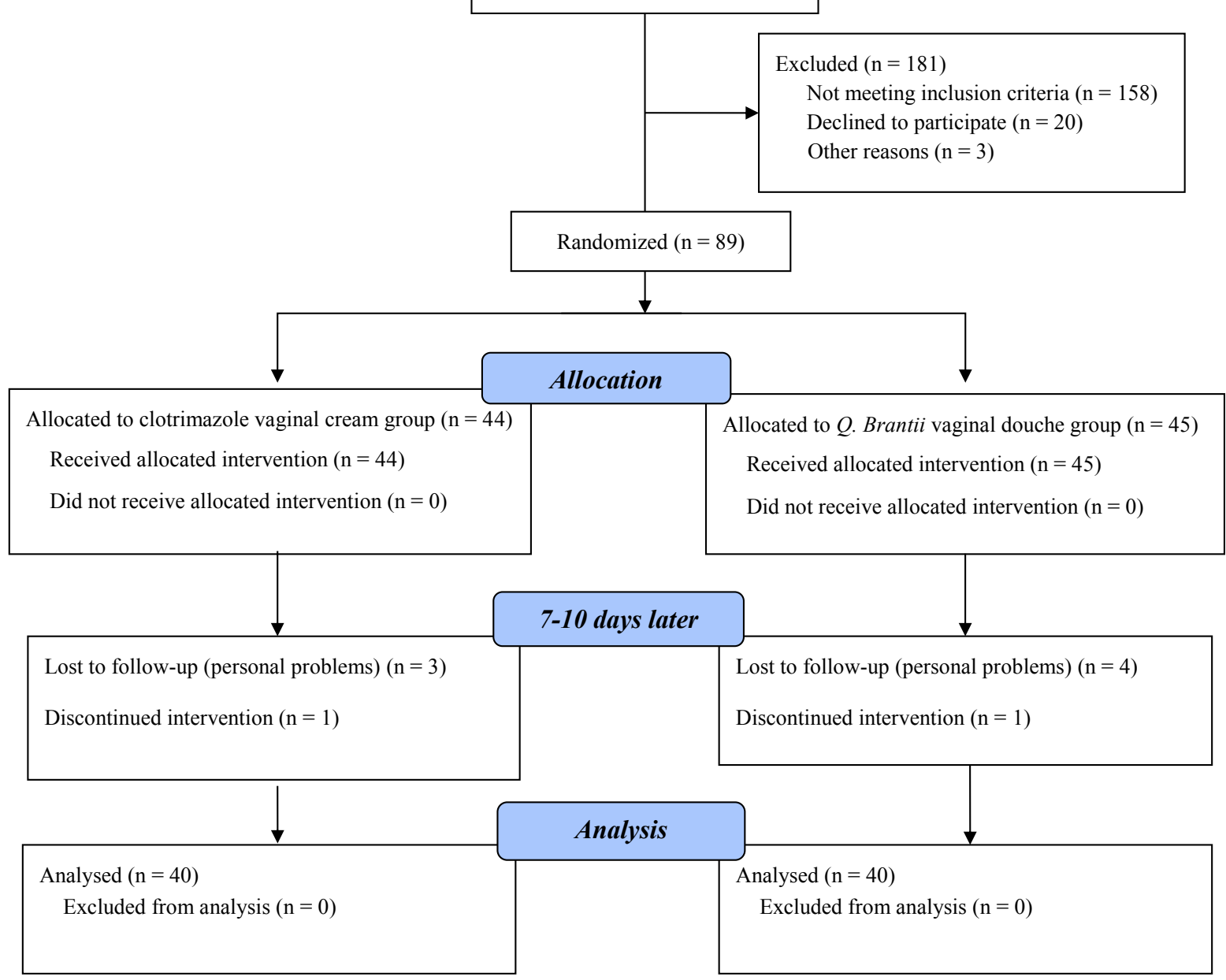

Figure 1 Flow diagram showing patients' recruitment and procedures ( CONSORT 2010) 
activities. Also, the alcoholic extract of Q. Brantii fruit has antimicrobial activities on Staphylococcus aureus, Staphylococcus epidermidis, and Escherichia coli [30].

In this research, vaginal candidiasis was significantly reduced in the regard of Candidiasis culture in Q. Brantii-treated group as compared to the group receiving clotrimazole vaginal cream. Improvements in clinical symptoms were not different among the groups. Khosravi et al. [31] investigated the therapeutic effects of Zataria multiflora cream and clotrimazole cream on acute vaginal candidiasis. They reported Zataria multiflora cream $(0.1 \%)$ to alleviate erythema and satellite vulvar lesions, vulvar and vaginal edemas and vulvovaginal excoriations in patients [31].

Antibacterial activities are involved in a complex mechanism like inhibitions of cell wall metabolism and synthesis, as well as cell memberane, nucleic acid, and protein [31]. Since having phenolic components, Q. Brantii fruit appears to induce a toxic effect against pathogens by reacting with sulfhydryl groups or more nonspecifically interacting with proteins through oxidized compounds [32]. Also, it is probable that the levels of phenolic compounds in $Q$. Brantii fruit enhance in response to pathogen infection [33]. Furthermore, linalool, geraniol, and terpinen-4-ol compounds as the representatives of oxygenated monoterpenes have been found to show the strongest antifungal activities against Candida albicans by deforming its cell wall membrane [34]. This pathogen impresses its effect by binding to host cells and inducing morphological changes in them through proteinase and phospholipase secretions [35]. Nonetheless, merit studies are required to clarify the pharmacological properties and mechanisms of phenolic compounds in Q. Brantii fruit extract. It has been reported that terpenes, phenols, carvacrol, flavonoids, eugenol, thymonin, and rosmarinic acids reduce the virulence properties of Candida albicans [36]. Moreover, furocoumarins, which are available in $Q$. Brantii, inhibit the growth of bacteria by interacting with the bacterial DNA $[37,38]$.Overall, terpenes and flavonoids (natural phenols) constitute the active antifungal compounds of essential oils. It seems that their antifungal or antibacterial mechanisms are related to those of other compounds [39]. Moreover, the antimicrobial activities of essential oils are dependent on their main components and synergistic or antagonistic effects [40].

\section{Conclusion}

In conclusion, the new findings of this study revealed that the bioactive substances existing in the inner stratum of $Q$. Brantii fruit have anti-fungal activities against vaginal candidiasis. The results of this research suggested almost similar therapeutic effects of the vaginal douche of Inner Stratum of $Q$. Brantii and clotrimazole vaginal cream. However, considering the limitation of the study such as unknown sensitivity to the new treatment, more studies are needed to determine the vaginal douche effect of $Q$. Brantiii in clinical trials.

\section{Acknowledgment}

We are extremely grateful to all the patients who participated in the study. This study was approved by the Shiraz University of Medical Sciences' research ethics committee (CT-92 6886). This research received its complete financial support from Shiraz University of Medical Sciences.

\section{Conflict of interest}

The authors declare that there are no conflicts of interest.

\section{References}

1. Weissenbacher T, Witkin SS, Ledger WJ, Tolbert V, Gingelmaier A, Scholz C, et al. Relationship between clinical diagnosis of recurrent vulvovaginal candidiasis and detection of Candida species by culture and polymerase chain reaction. Archives of gynecology and obstetrics. 2009;279(2):125-129.

2. Gonçalves B, Ferreira C, Alves CT, Henriques M, Azeredo J, Silva S. Vulvovaginal candidiasis: Epidemiology, microbiology and risk factors. Critical reviews in microbiology. 2016;42(6):905-927.

3. Bahadoran P, Rokni FK, Fahami F. Investigating the therapeutic effect of vaginal cream containing garlic and thyme compared to clotrimazole cream for the treatment of mycotic vaginitis. Iranian journal of nursing and midwifery research. 2010;15(Suppl1):343.

4. Akbarzdeh M, Bonyadpour B, Pakshir K. Causes and clinical symptoms of vaginal candidiasis in patients referring to selective clinics of Shiraz University of Medical Sciences (2009). Arak Medical University Journal. 2010;13(3).

5. Heng LS, Yatsuya H, Morita S, Sakamoto J. Vaginal douching in Cambodian women: its prevalence and association with vaginal candidiasis. Journal of epidemiology. 2010;20(1):70-76.

6. Zaki M, Begum W, Roqaiya M. Efficacy of Amla (Emblica officinanis) and Shibe yamani (potash alum) in the management of Candida vaginitis: a randomized standard controlled trial. International Journal of Reproduction, Contraception, Obstetrics and Gynecology. 2017;5(5):1601-1606.

7. Nazeri M, Mesdaghinia E, Moravej SAR, Atabakhshiyan R, Soleymani F. Prevalence of vulvovaginal candidiasis and frequency of candida species in women. Journal of Mazandaran University of Medical Sciences. 2012;21(86):254-262.

8. Ahmad A, Khan AU. Prevalence of Candida species and potential risk factors for vulvovaginal candidiasis in Aligarh, India. European journal of obstetrics \& gynecology and reproductive biology. 2009;144(1):68-71.

9. Cassone A. Vulvovaginal Candida albicans infections: pathogenesis, immunity and vaccine prospects. BJOG: An International Journal of Obstetrics \& Gynaecology. 2015;122(6):785-794.

10. Szigeti G, Sedaghati E, Mahmoudabadi AZ, Naseri A, Kocsube S, Vágvölgyi C, et al. Species assignment and antifungal susceptibilities of black aspergilli re- 
covered from otomycosis cases in Iran. Mycoses. 2012;55(4):333-338.

11. Mahmoudabadi AZ, Najafyan M, Moghimipour E, Alwanian M, Seifi Z. Lamisil versus clotrimazole in the treatment of vulvovaginal candidiasis. Iranian journal of microbiology. 2013;5(1):86.

12. Sekhavat L, Tabatabaii A, Tezerjani FZ. Oral fluconazole $150 \mathrm{mg}$ single dose versus intra-vaginal clotrimazole treatment of acute vulvovaginal candidiasis. Journal of infection and public health. 2011;4(4):195-199.

13. Crowley P, Gallagher H. Clotrimazole as a pharmaceutical: past, present and future. Journal of applied microbiology. 2014;117(3):611-617.

14. Artimani T, Shabanian S, Heidari-Soureshjani S, Asadi-Samani M, Luther T, Branch S. A REVIEW OF IRANIAN MEDICINAL PLANTS WITH TERATOGENIC AND ABORTION-INDUCING SIDE EFFECTS. INTERNATIONAL JOURNAL OF PHARMACEUTICAL SCIENCES AND RESEARCH. 2017;8(6):2372-2377.

15. Piddock LJ. The crisis of no new antibiotics-what is the way forward? The Lancet infectious diseases. 2012;12(3):249-253.

16. Seth AK, Geringer MR, Hong SJ, Leung KP, Mustoe TA, Galiano RD. In vivo modeling of biofilm-infected wounds: a review. Journal of surgical research. 2012;178(1):330-338.

17. Orhan I, Aslan M, Sener B, Kaiser M, Tasdemir D. In vitro antiprotozoal activity of the lipophilic extracts of different parts of Turkish Pistacia vera L. Phytomedicine. 2006;13(9):735-739.

18. Kokanova-Nedialkova Z, Nedialkov PT, Nikolov SD. The genus Chenopodium: phytochemistry, ethnopharmacology and pharmacology. Pharmacognosy Reviews. 2009;3(6):280.

19. Nabavizadeh MR, Zargaran A, Moazami F, Askari F, Sahebi S, Farhadpoor A, et al. Comparison of the Hemostatic Activity of Quercus persica Jaub. \& Spach. (Oak) With Ferric Sulfate in Bony Crypts. Journal of evidence-based complementary \& alternative medicine. 2016;21(1):34-38.

20. Popović BM, Štajner D, Ždero R, Orlović S, Galić Z. Antioxidant characterization of oak extracts combining spectrophotometric assays and chemometrics. The Scientific World Journal; 2013.

21. Feili M, Panahi J, Havasian MR. IN VITRO INHIBITORY EFFECT OF ALCOHOLIC EXTRACT OF INNER STRATUM OF OAK FRUIT (JAFT) ON CANDIDA ALBICANS. Iranian Journal of Public Health. 2014;43(2):277.

22. Pendharkar S, Brandsborg E, Hammarström L, Marcotte H, Larsson PG. Vaginal colonisation by probiotic lactobacilli and clinical outcome in women conventionally treated for bacterial vaginosis and yeast infection. BMC infectious diseases. 2015;15(1):255.

23. Hemmati A, Arzi A, Amin M. Effect of Achillea millefolium extract in wound healing of rabbit. Journal of Natural Remedies. 2002;2(2):164-167.

24. Peixoto F, Camargos A, Duarte G, Linhares I, Bahamondes L, Petracco A. Efficacy and tolerance of metronidazole and miconazole nitrate in treatment of vaginitis. International Journal of Gynecology \& Obstetrics. 2008;102(3):287-292.
25. Goldenberg RL, Mwatha A, Read JS, Adeniyi-Jones S, Sinkala M, Msmanga G, et al. The HPTN 024 Study: the efficacy of antibiotics to prevent chorioamnionitis and preterm birth. American journal of obstetrics and gynecology. 2006;194(3):650-661.

26. Roosens L, Cornelis C, D'Hollander W, Bervoets L, Reynders H, Van Campenhout K, et al. Exposure of the Flemish population to brominated flame retardants: Model and risk assessment. Environment International. 2010;36(4):368-376.

27. Roozegar MA, Panahi J, Havasian MR, Pakzad I. Studying the inhibitory effect of Alcoholic extract of inner Stratum of Oak Fruit (jaft) and hydro alcoholic extract of Summer bulb on Acinetobacter in vitro. International Research Journal of Biological Sciences; 2014.

28. Hashempur MH, Homayouni K, Ashraf A, Salehi A, Taghizadeh M, Heydari M. Effect of Linum usitatissimum L.(linseed) oil on mild and moderate carpal tunnel syndrome: a randomized, double-blind, placebo-controlled clinical trial. DARU Journal of Pharmaceutical Sciences. 2014;22(1):43.

29. Akram $^{1}$ E, Masoud K, Vahid N. Evaluation of the antibacterial and wound healing activity of Quercus persica. Journal of Basic \& Applied Sciences. 2012;8(1):118123.

30. Hojjati Bonab Z, Nikkhah E. Evaluation of antioxidant and antibacterial effect of methanolic extract from thyme (Thymus vulgar), senna (Cassia angustifolia) and licorice (Glycyrrhiza glabra). Daneshvar. 2012;19(100):57-66.

31. Khosravi AR, Eslami AR, Shokri H, Kashanian M. Zataria multiflora cream for the treatment of acute vaginal candidiasis. International Journal of Gynecology \& Obstetrics. 2008;101(2):201-202.

32. Dambolena J, López A, Cánepa M, Theumer M, Zygadlo J, Rubinstein $\mathrm{H}$. Inhibitory effect of cyclic terpenes (limonene, menthol, menthone and thymol) on Fusarium verticillioides MRC 826 growth and fumonisin B1 biosynthesis. Toxicon. 2008;51(1):37-44.

33. Bais HP, Walker TS, Schweizer HP, Vivanco JM. Root specific elicitation and antimicrobial activity of rosmarinic acid in hairy root cultures of Ocimum basilicum. Plant Physiology and Biochemistry. 2002; 40(11):983-995.

34. Marcos-Arias C, Eraso E, Quindós G, Madariaga L. In vitro activities of natural products against oral Candida isolates from denture wearers. BMC Complementary and Alternative Medicine. 2011;11(1):119.

35. Haynes K. Virulence in Candida species. Trends in microbiology. 2001;9(12):591-596.

36. Adwan G, Salameh Y, Adwan K, Barakat A. Assessment of antifungal activity of herbal and conventional toothpastes against clinical isolates of Candida albicans. Asian Pacific journal of tropical biomedicine. 2012;2(5):375-379.

37. Issa S, Mehdi H, Sakine S, Shoele J. Antimicrobial effects of Quercus brantii fruits on bacterial pathogens. Jundishapur Journal of Microbiology. 2012;2012(3, Summer):465-469.

38. Safary A, Motamedi H, Maleki S, Seyyednejad SM. A preliminary study on the antibacterial activity of Quer- 
cus brantii against bacterial pathogens, particularly enteric pathogens. International Journal of Botany. 2009;5(2):176-180.

39. Hossain MA, Shah MD, Sang SV, Sakari M. Chemical composition and antibacterial properties of the essential oils and crude extracts of Merremia borneensis. Journal of King Saud University-Science. 2012; 24(3):243-249.

40. Mahboubi M, Bokaee S, Dehdashti H, Feizabadi MM. Antimicrobial activity of Mentha piperitae, Zhumeria majdae, Ziziphora tenuior oils on ESBLs producing isolates of Klebsiella pneumonia. Biharean Biol. 2012;6:59. 